\title{
Morfologias e manifestações clínicas da ponte miocárdica: uma revisão narrativa
}

\author{
Myocardial bridge morphologies and clinical manifestations: a narrative review
}

Morfologías del puente miocárdico y manifestaciones clínicas: una revisión narrativa

Laerte de Paiva Viana Filho ${ }^{1 *}$, Williana Garcia Braga1, Diana Antoniazzi de Sá Ribeiro², Fernando Lucas Santos Magalhães², Israel Farias de Souza², Maísa Aparecida Marques Araújo², Sara Custódio Martins², Thaila Martins Silva².

\section{RESUMO}

Objetivo: Discorrer a respeito da ponte miocárdica $(\mathrm{PM})$, com destaque às descrições morfológicas, as implicações clínicas, o diagnóstico e o seu tratamento. Revisão bibliográfica: A PM acomete majoritariamente a artéria coronária descendente anterior esquerda. Na clínica, observa-se uma minoria sintomática, mas deve ser considerada em pacientes com baixo risco para doença coronariana aguda e com sintomas de angina instável ou estável. As principais implicações clínicas relacionadas à PM são aterosclerose e isquemia miocárdica. Para o diagnóstico, a tomografia computadorizada multislice das coronárias e a angiografia coronária convencional são os principais exames utilizados, em que avaliam a compressão da artéria coronária por PM durante a sístole. Há uma discrepância significativa entre a prevalência dos exames in vivo e na autópsia. Como tratamento, a intervenção medicamentosa, cirúrgica e/ou percutânea via stent podem ser realizadas, de acordo com o quadro clínico de cada paciente. Considerações finais: A PM permanece um tema controverso, visto sua elevada parcela assintomática, mas de suma importância para a cardiologia clínica e intervencionista nos casos sintomáticos.

Palavras-chave: Ponte miocárdica, Aterosclerose, Isquemia miocárdica, Circulação coronária.

\begin{abstract}
Objective: To discuss the myocardial bridge (MB), with emphasis on the morphological descriptions, the clinical implications, the diagnosis and its treatment. Literature review: MB mainly affects the left anterior descending coronary artery. In the clinic, a symptomatic minority is observed, but it should be considered in patients at low risk for acute coronary disease and with symptoms of unstable or stable angina. The main clinical implications related to MB are atherosclerosis and myocardial ischemia. For diagnosis, multislice computed tomography of the coronary arteries and conventional coronary angiography are the main tests used, in which they evaluate the compression of the coronary artery by MB during systole. There is a significant discrepancy between the prevalence of in vivo examinations and the autopsy. As treatment, drug, surgical and / or percutaneous stent intervention can be performed, according to the clinical condition of each patient. Final considerations: MB remains a controversial topic, given its high asymptomatic portion, but of paramount importance for clinical and interventional cardiology in symptomatic cases.
\end{abstract}

Keywords: Myocardial bridging, Atherosclerosis, Myocardial ischemia, Coronary circulation.

\section{RESUMEN}

Objetivo: Discutir el puente miocárdico (PM), con énfasis en las descripciones morfológicas, las implicaciones clínicas, el diagnóstico y su tratamiento. Revisión bibliográfica: PM afecta principalmente la arteria coronaria descendente anterior izquierda. En la clínica, se observa una minoría sintomática, pero debe considerarse en pacientes con bajo riesgo de enfermedad coronaria aguda y con síntomas de angina inestable o estable. Las principales implicaciones clínicas relacionadas con la MP son la aterosclerosis y la isquemia miocárdica. Para el diagnóstico, las principales pruebas utilizadas son la tomografía computarizada multicorte de las arterias coronarias y la angiografía coronaria convencional, en las que evalúan la compresión de la arteria coronaria por PM durante la sístole. Existe una discrepancia significativa entre la prevalencia de los exámenes in vivo y la autopsia. Como tratamiento, se puede realizar una intervención farmacológica, quirúrgica y / o con stent

\footnotetext{
1 Pontifícia Universidade Católica de Minas Gerais (PUC-MG), Betim - MG. *E-mail: laerteviana07@gmail.com 2 Universidade Federal de Ouro Preto (UFOP-MG), Ouro Preto - MG.
} 
percutáneo, de acuerdo con el estado clínico de cada paciente. Consideraciones finales: PM sigue siendo un tema controvertido, dada su alta porción asintomática, pero de suma importancia para la cardiología clínica e intervencionista en casos sintomáticos.

Palabras clave: Puente miocárdico, Aterosclerosis, Isquemia miocárdica, Circulación coronaria.

\section{INTRODUÇÃO}

As artérias coronárias e seus principais ramos, responsáveis pela vascularização do coração, estão localizadas normalmente em um curso subepicárdico. Entretanto, existe uma anomalia congênita em que segmentos de uma ou mais artérias coronárias são sobrepostos por uma faixa de músculo cardíaco: a ponte miocárdica $(\mathrm{PM})$. Também conhecida como artéria coronária intramural, artéria coronária mural e artéria intramural, a PM foi primeiramente descrita em 1737 por Reyman em uma autópsia e, posteriormente, teve sua primeira descrição angiográfica in vivo em 1960 por Porstmann e Iwig. As pontes miocárdicas são mais comumente localizadas no segmento médio da artéria coronária descendente anterior esquerda, sendo que pode haver envolvimento de outras artérias, como a artéria circunflexa esquerda e a artéria coronária direita (LIMA VJ, et al., 2002; THEJ MJ, et al., 2012; YUAN SM, 2016).

Fisiologicamente, os vasos acometidos pela ponte miocárdica sofrem uma estenose durante a sístole, que ocasiona um aumento do fluxo retrógrado de sangue nessa fase do ciclo cardíaco. Mesmo que a PM cause, a princípio, a compressão do vaso durante a sístole, as duas fases cardíacas são afetadas. Ademais, há um posterior aumento precoce na velocidade do fluxo do sangue na diástole, e um consequente retardo no relaxamento diastólico no segmento envolvido pela ponte (BARROS MV, et al., 2013; HOSTIUC S, et al., 2017). Embora a artéria coronária intramural seja considerada benigna por muitos autores, estudos a associam a várias condições clínicas, correlacionadas a um provável significado hemodinâmico da PM, como síndromes coronarianas agudas, espasmo coronário, arritmias, bloqueio da condução atrioventricular induzida pelo exercício, atordoamento cardíaco, morte súbita e, sobretudo, isquemia do miocárdio e aterosclerose (BARROS MV, et al., 2013; THEJ MJ, et al., 2012).

Atualmente, os principais métodos para o diagnóstico da PM in vivo são a angiografia coronária convencional e a tomografia computadorizada multislice das coronárias, enquanto a técnica para a detecção em cadáveres é a autópsia (BARROS MV, et al., 2013). Somado a isso, o seu impacto funcional é geralmente avaliado com ultrassom Doppler intracoronário, ultrassom intravascular e dispositivos de pressão intracoronariana (HOSTIUC S, 2011).

A real prevalência da PM ainda não está esclarecida, com perceptível discrepância nos dados documentados, sendo que na angiografia a taxa de acometimento varia de 0,5 a 12\%, enquanto na autópsia de 5,4 a $85,7 \%$. Além disso, a diferença de prevalência entre os gêneros não é estatisticamente significativa (ACUNÃ LE, et al., 2009). O subdiagnóstico da PM ocorre em virtude de a variedade dos métodos utilizados possuir acurácia diferente e devido à maioria dos pacientes ser assintomática, o que pode ser explicado pela angiogênese de ramos coronarianos na região proximal à $P M$, favorecendo a retomada da irrigação mesmo com essa variação anatômica (ACUNÃ LE, et al., 2009; BARROS MV, et al., 2013; PEREIRA AB, et al., 2010).

O objetivo deste trabalho foi produzir uma revisão literária a respeito da ponte miocárdica, destacando as descrições morfológicas, as manifestações clínicas, em especial a aterosclerose e a isquemia do miocárdio, o diagnóstico e o tratamento.

\section{REVISÃO BIBLIOGRÁFICA}

\section{Descrições morfológicas}

A descrição morfológica das pontes miocárdicas é apresentada de forma heterogênea na literatura científica. Isso ocorre devido aos limites técnicos dos métodos utilizados para estudá-las. Mesmo com essa discordância, existem estudos que obtiveram dados importantes para a compreensão dessa anomalia (HOSTIUC S, et al., 2018). 
É apontado que a ponte miocárdica acomete majoritariamente a artéria coronária descendente anterior esquerda (DAE), porém é encontrada também em outras artérias coronárias. Isso pode ser evidenciado por meio dos dados, em que: nos exames, incluindo in vivo e autópsia, a DAE tem uma prevalência de 0,82 e na autópsia exclusivamente, de 0,63. Além disso, os segmentos da DAE possuem diferentes índices de acometimento, sendo que o segmento médio é o mais afetado, com predomínio de 0,72 , seguido pelo seguimento distal $(0,23)$ e o segmento proximal $(0,04)$. Assim como a DAE, há uma variação estatística entre a totalidade dos exames e a autópsia isolada, respectivamente, nas outras artérias coronárias afetadas: artéria intermediária $(0,01$ e 0,01$)$, artéria circunflexa $(0,03$ e 0,07$)$, artérias marginais $(0,04$ e 0,05$)$, artérias diagonais $(0,05$ e 0,08$)$ e artéria coronária direita incluindo a artéria interventricular posterior $(0,04$ e 0,16$)$ (HOSTIUC S, et al., 2018).

A gravidade e os efeitos das pontes miocárdicas são melhor definidos pela fisiologia do que pela anatomia (GOULD KL e JOHNSON NP, 2015), contudo há hipóteses de que as características anatômicas da PM podem estar relacionadas com implicações clínicas. Nesse sentido, as artérias coronárias murais foram classificadas em benigna ou patológica de acordo com os critérios de comprimento e de profundidade intramiocárdica (DE GIORGIO F, et al., 2014). A anomalia é considerada patológica quando o segmento tunelizado tem de 20 a $30 \mathrm{~mm}$ de comprimento e 2 a $3 \mathrm{~mm}$ de profundidade (BASSO C, et al., 2009).

\section{Manifestações clínicas}

A relevância clínica das pontes miocárdicas é heterogênea, e a minoria dos pacientes é sintomática, podendo apresentar isquemia miocárdica, síndromes coronárias agudas, espasmos coronários, disritmias induzidas pelo exercício (como taquicardia supraventricular, taquicardia ventricular ou bloqueio atrioventricular), atordoamento miocárdico, disfunção ventricular transitória, síncope ou até morte súbita (LIMA VJ, et al., 2002; HOSTIUC S, 2011; PEREIRA AB, et al., 2010; YUAN SM, 2016).

A alta incidência de ramos pré-ponte (RPP) no segmento acometido pela PM pode agir como mecanismo compensatório, conhecido por angiogênese, devido à irrigação da região adjacente. Essa hipótese explicaria a ausência de sintomatologia em alguns pacientes (ACUNÃ LE, et al., 2009). Ainda que a malformação esteja presente desde o nascimento, os sintomas em geral surgem a partir da terceira década de vida, com média de 45,9 anos. A expressão tardia dos sintomas pode ser justificada por meio de três fatores: o aumento da tensão sistólica da parede miocárdica em consequência do crescimento do coração; o aumento da pressão diastólica final do ventrículo esquerdo; e as relações dos sintomas com a hipertensão arterial e a eventual diminuição do fluxo coronário por eventos ateroscleróticos que ocorrem mais tardiamente (PEREIRA AB, et al., 2010).

Apesar de a PM geralmente apresentar um curso assintomático e com bom prognóstico, estudos demonstraram sua associação com quadros clínicos (BARROS C, et al., 2013). Dessa forma, o surgimento dos sintomas pode ocorrer com dois mecanismos distintos. Um acontece em razão da estimulação e aceleração da aterosclerose no segmento proximal à ponte do miocárdio. O outro, pela contração das fibras da ponte miocárdica e compressão direta do segmento tunelizado (YUAN SM, 2016).

Na clínica, a suspeita de ponte miocárdica é verificada principalmente em pacientes com baixo risco para doença coronariana aguda (DAC) e com sintoma de angina instável ou estável. Observa-se que não há correlação entre a sintomatologia e o grau de estreitamento angiográfico na literatura. Entretanto, a espessura, a localização, o número e o comprimento das PMs possuem vínculo direto com a expressão dos sintomas (PEREIRA AB, et al., 2010).

\section{Aterosclerose}

Durante a elaboração do presente trabalho, foram observadas divergências de análise a respeito da associação entre a ponte miocárdica e a aterosclerose. Existem estudos que apontam que essa correlação não é tão significativa quando comparada com os fatores de risco cardiovasculares tradicionais, não sendo detectadas diferenças expressivas relacionadas ao acometimento da aterosclerose entre pacientes portadores de PM e sem a anomalia (SUN JL, et al., 2013). Por outro lado, hipóteses defendem que os segmentos coronarianos afetados pela ponte miocárdica expõem maior tendência para formação de placas ateroscleróticas em relação aos vasos epicárdicos (BARROS C, et al., 2013). 
Para definir aterosclerose, foi utilizado como parâmetro a presença de estruturas maiores ou iguais a 1 $\mathrm{mm}^{2}$ no interior e/ou adjacente à luz da artéria coronária, em que a placa aterosclerótica foi classificada como obstrutiva quando $50 \%$ de estreitamento luminal fosse observado (BARROS C, et al., 2013). A incidência do processo aterosclerótico em pacientes com PM ocorre particularmente nos segmentos proximal e distal, com prevalência preponderante no primeiro. Entretanto, a expressão de aterosclerose também pode ser encontrada, de maneira minoritária, no segmento tunelizado (BARROS C, et al., 2013; ENHOS A, et al., 2019).

Uma hipótese que sustenta o maior acometimento aterosclerótico no segmento proximal da PM é a perturbação do fluxo sanguíneo intracoronariano, que ocorre devido às forças hemodinâmicas presentes anteriormente à entrada do segmento intramural. Essa irregularidade modifica a produção de substâncias vasoativas das células endoteliais, como óxido-nítrico, endotelina-1 e angiotensina, o que altera o potencial trombogênico, a regulação do fluxo sanguíneo e o tônus vascular (BARROS C, et al., 2013; ENHOS A, et al., 2019). Somado a isso, o elevado estresse mural proximal a PM também pode contribuir para a precipitação de placa aterosclerótica em virtude das lesões na túnica íntima (BARROS C, et al., 2013).

É sabido que a aterosclerose é um processo inflamatório, sendo medida através da razão de monócitos para lipoproteínas de alta densidade (MHR - sigla em inglês). Esse parâmetro é funcional tendo em vista que monócitos diferenciados em macrófagos atuam na inflamação crônica e em doenças cardiovasculares, modulando citosinas inflamatórias. O colesterol HDL, por sua vez, possui propriedades anti-inflamatórias, antioxidantes e antitrombóticas, fazendo com que essa molécula tenha a capacidade de mitigar a expressão endotelial de moléculas de adesão, o que reduz o recrutamento de monócitos para a parede arterial, inibindo a inflamação. Além do mais, essa lipoproteína de alta densidade minimiza os efeitos pró-inflamatórios e próoxidantes dos monócitos, interferindo na migração de macrófagos e na oxidação de lipoproteínas de baixa densidade (LDL). Isso configura um fator relevante, tendo em vista que essa função antioxidante diminui a progressão da doença aterosclerótica (ENHOS A, et al., 2019).

Na literatura, foi encontrada uma significância entre pacientes com ponte miocárdica e os níveis elevados de MHR visto que a disfunção endotelial, o processo inflamatório e a expressão elevada de agentes vasoativos foram mecanismos ateroscleróticos causados pela PM. Por essa ótica, após o início da inflamação, os monócitos agravaram essas vias inflamatórias, dando progressão à aterosclerose. O HDL, que seria responsável pela diminuição desses mecanismos inflamatórios, encontrou-se em níveis baixos nos pacientes com PM, ao passo que os monócitos estavam elevados refletindo em uma alta taxa de MHR, o que foi associado como marcador prognóstico de doenças cardiovasculares. Em suma, foi evidenciado que a elevada MHR poderia demonstrar tanto a arteriosclerose sistêmica quanto a arteriosclerose local, concluindo que modificações locais no segmento proximal da PM poderiam começar mais cedo do que as alterações sistêmicas (ENHOS A, et al., 2019).

\section{Isquemia miocárdica}

A isquemia miocárdica (IM) é definida como a falta de suprimento sanguíneo, o qual é responsável pela nutrição e oxigenação do tecido cardíaco, em comparação com a demanda do local (YU M, et al., 2015). Nesse aspecto, estudos que apontam hipóteses de proporcionalidade direta entre ponte miocárdica e IM foram encontrados nas bibliografias de referência (HOSTIUC S, et al., 2017). O exame que é utilizado para avaliar essa patologia em PM é a cintilografia do miocárdio (LIM JW, et al., 2017).

Existem dois processos fisiológicos que estão associados à IM: o primeiro é a pressão da ponte miocárdica sobre a artéria intramural, em que ocorre o estreitamento do vaso por PM durante a sístole. Devido a essa oclusão, o mecanismo compensatório desenvolvido pelo coração para elevar o fluxo sanguíneo ao nível de demanda é o aumento da pressão diastólica, que é diretamente proporcional com o tempo de duração de compressão coronariana sistólica. Contudo, essa elevação não é suficiente para suprir a irrigação sanguínea local, tendo como consequência um atraso de relaxamento diastólico precoce, o que pode ocasionar a isquemia (HOSTIUC S, et al., 2017). Nota-se que o aumento da frequência cardíaca intensifica a gravidade da IM, pois a fase diastólica é reduzida. Isso faz com que a demanda necessária de sangue não seja atingida, uma vez que em casos de PM o preenchimento diastólico já é prejudicado. Com o aumento da frequência cardíaca, esse suprimento ficará ainda mais debilitado, provocando maior susceptibilidade à IM (SUN JL, et al., 2013). 
O segundo é a aterosclerose proximal ao segmento em ponte, causada por alterações de células endoteliais nessa região e consequente junção de placa aterosclerótica, que aumenta a estenose coronariana (BARROS C, et al., 2013). Com essa diminuição de lúmen, há redução do suprimento sanguíneo, levando à isquemia (BARROS C, et al., 2013). Constata-se que o vasoespasmo em segmento aterosclerado pode, também, levar à isquemia coronariana (HONG H, et al., 2014).

Algumas situações podem potencializar a prevalência da isquemia em pacientes com ponte miocárdica, como a ativação simpática e a localização da PM. O estresse e os exercícios físicos promovem ativação simpática e, com isso, elevam a frequência cardíaca e a força de contração, responsáveis pelo aumento da probabilidade de IM em indivíduos com PM. Além disso, caso a PM esteja localizada na extremidade mais proximal do vaso, há uma maior chance de acometimento por IM, visto que essa região é mais passível de ser acometida por aterosclerose (DE GIORGIO F, et al., 2014).

\section{Diagnóstico}

O diagnóstico para PM possui um valor essencial nos seus significados anatômico e clínico, pois a partir dele é possível a sua identificação, como também de outras possíveis patologias, como aterosclerose e isquemia do miocárdio. A recomendação para a realização de exames diagnósticos para essa anomalia deve ser considerada a partir da terceira década de vida do paciente que apresenta sintomas de dor anginosa, mas sem condições de risco para outras patologias do coração (PEREIRA AB, et al., 2010). Ademais, o princípio de análise no qual esses exames estão pautados é a avaliação da compressão que a artéria coronária sofre pela PM durante a sístole e sua liberação completa ou parcial durante a diástole (ROVAI D, et al., 2015). Por ser uma patologia majoritariamente assintomática, ela é subdiagnosticada in vivo (PEREIRA AB, et al., 2010). Para a investigação da PM, podem ser destacados os exames: angiografia coronária convencional e tomografia computadorizada multislice das coronárias (BARROS MV, et al., 2013).

A angiografia coronária convencional, também chamada de arteriografia coronária ou cineangiocoronariografia (CAG) é tido como o exame para o diagnóstico de pontes com maior profundidade $(>1 \mathrm{~mm}$ ) (BACHUR LF, et al., 2003). Contudo, a PM será diagnosticada como patológica quando o achado for (>2mm) (BASSO C, et al., 2009). Para essa análise, os principais parâmetros a serem considerados são: espessura e largura da PM, a posição correspondente da PM à artéria coronária mural e a presença de tecido adiposo ou conjuntivo ao redor da artéria coronária mural. Quando juntos, esses parâmetros detectam a PM por meio da identificação da compressão de segmento coronário, geralmente o ramo descendente anterior esquerdo, durante a sístole (SUN JL, et al., 2013).

Para avaliar o nível de compressão da artéria coronária, é observado o "efeito ordenha". Esse fenômeno é descrito como imagem ausente ou pouco desenvolvida na fase sistólica, devido à estenose do lúmen, e imagem luminal desenvolvida, clara e evidente, durante a fase diastólica. Diante disso, estudos padronizaram o nível de estenose durante a sístole da seguinte forma: grau I / 50\% (leve); grau II / 50-70\% (médio); grau III / 70\% (grave) (SUN JL, et al., 2013). Esse parâmetro se torna significativo quando ocorre uma diminuição de $\geq 70 \%$ no diâmetro luminal da artéria durante a sístole e a persistência dessa redução de $\geq 35 \%$ durante a diástole (YUAN SM, 2016).

Quando a realização desse exame se torna de difícil visualização são utilizados vasodilatadores, como o nitrato em forma de nitroglicerina, acetilcolina ou papaverina, que dilatam o lúmen do segmento intramiocárdico, evidenciando ainda mais a compressão sistólica. Isso pode ser visto em estudos comparativos os quais mostram que com a utilização do nitrato, a incidência média de PM nas coronariografias diagnósticas pode alcançar até $40 \%$ dos casos, e na ausência desse composto, a incidência situa-se em 2\% (SANTOS LD, et al., 2007).

Juntamente com a avaliação anatômica proporcionada pela CAG, são usados também outros métodos, como o ultrassom intracoronário (USIC), o ultrassom Doppler intracoronariano e os dispositivos de pressão intracoronariana, que avaliam a dinamicidade e a correlação com outras condições clínicas, a exemplo da formação de placa aterosclerótica. Em USIC, a PM foi definida como uma faixa ecolucente (halo) circundando parcialmente a artéria. A avaliação anatômica do USIC inclui o comprimento do segmento, a espessura do halo, localização e presença de ramificações que surgem no segmento em PM (YAMADA R, et al., 2016). 
A angiotomografia computadorizada multislice ou Angio TC é apontada como um exame seguro e confiável para a avaliação da PM, tendo em vista sua capacidade de identificar de forma clara e precisa todo o curso coronariano. Por meio dele, é possível obter a diferença de posição espacial e temporal de artérias coronárias interarteriais com caminho intramural daquelas que não fazem esse percurso (ATTILI A, et al., 2013).

Além disso, ao analisar hemodinamicamente as artérias, tem-se uma clara detecção da parede dos vasos e do seu diâmetro, o que permite observar se há presença de placas ou risco para o desenvolvimento da aterosclerose. Seu principal diferencial em relação a cineangiocoronariografia é o fato de não precisar da compressão sistólica para obter a avaliação, sendo um método não-invasivo (DOS SANTOS KER W, et al., 2017).

A relação da Angio TC com outros exames, como o ecocardiograma e a ressonância magnética é fundamental, tendo em vista que são complementares para um diagnóstico diferencial de patologias (ATTILI A, et al., 2013). O ecocardiograma obtém informações mais precisas de pacientes com PM, sendo possível medir o grau da lesão com medições longitudinais, circunferenciais e radiais (YUAN SM, 2016). A ressonância magnética, por sua vez, tem um papel essencial no diagnóstico infanto-juvenil, reconhecendo e avaliando a função cardíaca por meio da análise do curso das artérias em um cenário que o ecocardiograma não é tão preciso (ATTILI A, et al., 2013; HOSTIUC S, 2011).

A maior prevalência de ponte miocárdica foi relatada em autópsia, seguida pela Angio TC e pela CAG, com valores significativamente discrepantes (HOSTIUC S, et al., 2018). Essa divergência pode ser justificada pela ausência de sintomatologia in vivo e pela angiotomografia não demandar compressão sistólica, o que aprimora a visualização anatômica dessa condição. Outro fator de suma importância é que a CAG não tem acurácia para identificar as PMs finas e superficiais, pois não produzem o "efeito ordenha" (HOSTIUC S, et al., 2018; LI Y, et al., 2017; PEREIRA AB, et al., 2010; YUAN SM, 2016).

\section{Tratamento}

O tratamento para ponte miocárdica é feito de acordo com o quadro clínico de cada paciente, podendo ser intervenção medicamentosa, cirúrgica e/ou percutânea via stent (ESTEVES V, et al., 2010). Essas terapêuticas, apesar de terem abordagens distintas, objetivam melhorar o fluxo sanguíneo coronariano em pacientes com artéria coronária intramural (BARROS MV, et al., 2013).

Como tratamento inicial, é recomendada a terapia medicamentosa, que é composta por agentes ionotrópicos e cronotópicos negativos - como os betabloqueadores e os antagonistas dos canais de cálcio e os agentes antiplaquetários. Os bloqueadores de receptores adrenérgicos, também denominados betabloqueadores, geram redução da contratilidade e da frequência cardíaca, e aumento do tempo diastólico e da compressão sistólica do vaso, agindo na melhoria da angina e dos sinais de isquemia. Os antagonistas de cálcio, por sua vez, aparecem como alternativa quando há contraindicação dos betabloqueadores, e são o tratamento de primeira linha no caso de vasoespasmo coronariano. Nas condições em que há risco de aterosclerose, a terapia antiplaquetária é indicada (PEREIRA AB, et al., 2010; YUAN SM, 2016).

Nota-se que a nitroglicerina, agente vasodilatador, ao melhorar a contratilidade cardíaca, intensifica 0 estreitamento sistólico da coronária intramural, exacerbando os sintomas associados à PM. Dessa forma, esse fármaco deve ser prescrito com cautela (YUAN SM, 2016).

Outra terapêutica é a implantação percutânea de Stent na porção arterial intramural, cujo intuito é manter o lúmen aberto e, dessa forma, aliviar a compressão sistólica sobre a coronária e normalizar o fluxo diastólico. Essa intervenção reduz a angina e outros sintomas, sendo indica para pacientes sintomáticos persistentes. No entanto, foi relatado, em estudos, casos em que a endoprótese expansível apresentou estenose após determinado período, demandando novas intervenções e limitando seu uso (PEREIRA AB, et al., 2010).

A intervenção cirúrgica também é uma alternativa indicada para pacientes com sintomas persistentes que não obtiveram êxito com o tratamento farmacológico. Via miotomia, ocorre a descompressão cirúrgica da artéria intramural, o que alivia os sintomas de angina e isquemia. Esse procedimento pode envolver, no entanto, algumas complicações, como a perfuração da parede e o aneurisma ventricular. Já na cirurgia de revascularização do miocárdio, uma outra possibilidade, há a realização de anastomoses da artéria mamária 
interna na DAE, técnica altamente invasiva e que envolve maior tempo de recuperação, não configurando um tratamento prioritário (PEREIRA AB, et al., 2010; SUN JL, et al., 2013). Por fim, apesar de as abordagens cirúrgicas terem tido ampla utilização nos últimos anos, atualmente, elas têm sido substituídas pela intervenção percutânea (PEREIRA AB, et al., 2010).

\section{CONSIDERAÇÕES FINAIS}

Embora seja um achado anatômico que normalmente não gere sintomas, a ponte miocárdica pode causar implicações, como: angina, aterosclerose, isquemia do miocárdio, entre outras. A PM permanece, portanto, um assunto controverso, mas é de suma importância para a cardiologia clínica e intervencionista. Nessa perspectiva, a insuficiência de dados impossibilita a tomada de conclusões definitivas sobre a temática, sendo necessários estudos para a construção de uma propedêutica mais sistematizada em relação a ponte miocárdica.

\section{REFERÊNCIAS}

1. ACUNÃ L, et al. Descrição morfológica e implicações clínicas de pontes miocárdicas: um estudo anatômico em colombianos. Arquivos Brasileiros de Cardiologia, 2009; 92(4): 256-262.

2. ATTILI A, et al. Echocardiography and angiotomography of coronary images of variations in coronary anatomy and coronary abnormalities in athletic children: detection of coronary abnormalities that create a risk of sudden death. Echocardiography, 2013; 30(2): 225-233.

3. BACHUR LF, et al. Correlação Anatomoclínica, 2003; 81(6).

4. BARROS MV, et al. Avaliação da ponte miocárdica pela angiotomografia das coronárias. Rev. bras. ecocardiogr. imagem cardiovasc, 2013; 26(1): 8-15.

5. BASSO C, et al. Myocardial bridging, a frequent component of the hypertrophic cardiomyopathy phenotype, lacks systematic association with sudden cardiac death. Eur Heart J, 2009; 30: 1627-1634.

6. DE GIORGIO F, et al. Myocardial bridging and sudden cardiac death: Is the actual classification exhaustive? International Journal of Cardiology, 2014; 172(3): 2013-2014.

7. DOS SANTOS KER W, et al. Myocardial bridge and angiotomography of the coronary arteries: Perfusion under pharmacological stress. Arquivos Brasileiros de Cardiologia, 2017; 108(6): 572-575.

8. ENHOS A, et al. Assessment of the relationship between monocyte to high-density lipoprotein ratio and myocardial bridge. Arquivos Brasileiros de Cardiologia, 2019; 112(1): 12-17.

9. ESTEVES V, et al. Infarto agudo do miocárdio associado a ponte miocárdica. Rev. Bras. Cardiol., 2010; 18(4): 468472.

10. GOULD KL, JOHNSON NP. Myocardial bridges: Lessons in clinical coronary pathophysiology. JACC: Cardiovascular Imaging, 2015; 8(6): 705-709.

11. HONG $\mathrm{H}$, et al. Angiographically evident atherosclerotic stenosis associated with myocardial bridging and risk factors for the artery stenosis located proximally to myocardial bridging. Anadolu Kardiyoloji Dergisi, 2014; 14(1): 40-47.

12. HOSTIUC S, et al. Cardiovascular consequences of myocardial bridging: A meta-analysis and meta-regression. Scientific Reports, 2017; 7(1): 1-13.

13. HOSTIUC S, et al.. Morphological changes associated with hemodynamically significant myocardial bridges in sudden cardiac death. Thorac Cardiovasc Surg, 2011; 59(7): 393-398.

14. HOSTIUC S, et al. Myocardial Bridging: A Meta-Analysis of Prevalence. Journal of Forensic Sciences, 2018; 63(4): 1176-1185.

15. LI Y, et al. Non-invasive imaging of myocardial bridge by coronary computed tomography angiography: the value of transluminal attenuation gradient to predict significant dynamic compression. European Radiology, 2017; 27(5): 19711979.

16. LIM JW, et al. Myocardial CT perfusion imaging for pre-A nd postoperative evaluation of myocardial ischemia in a patient with myocardial bridging. Medicine (United States), 2017; 96(42): e8277

17. LIMA VJ, et al. Pontes de miocárdio e sua relação com o ramo interventricular anterior da artéria coronária esquerda. Arq. bras. cardiol, 2002; 79(3): 215-222.

18. PEREIRA AB, et al. Ponte miocárdica: evolução clínica e terapêutica. Arquivos Brasileiros de Cardiologia, 2010; 94(2): 188-194.

19. ROVAI D, et al. Myocardical bridging: a review with emphasis on electrocardiographic findings. Ann Noninvasive Electrocardiol, 2015; 20(2): 103-107. 
20. SANTOS LD, et al. Relato de Caso Ponte Miocárdica Multiarterial : Apresentações Clínica e Anatômica. Arq. Bras. Cardiol., 2007; 88(4) 73-75.

21. SUN JL, et al. Relationship Between Myocardial Bridging and Coronary Arteriosclerosis. Cell Biochemistry and Biophysics, 2013; 65(3): 485-489.

22. THEJ MJ, et al. Atherosclerosis and myocardial bridging: Not a benign combination. An autopsy case report. Journal of Cardiovascular Disease Research, 2012; 3(2): 176-178.

23. YAMADA R, et al. Functional versus anatomic assessment of myocardial bridging by intravascular ultrasound: Impact of arterial compression on proximal atherosclerotic plaque. Journal of the American Heart Association, 2016; 5(4): 19.

24. YU M, et al. Myocardia ischemia associated with a myocardial bridge with no significant atherosclerotic stenosis. BMC Cardiovascular Disorders, 2015; 15(1): 2-5.

25. YUAN SM. Myocardial bridging. Brazilian Journal of Cardiovascular Surgery, 2016; 31(1): 60-62. 